\title{
Mudanças na fertilidade de um Neossolo Regolítico após seis anos de adubação orgânica
}

\author{
Rômulo S. C. Menezes ${ }^{1} \&$ Tácio O. da Silva ${ }^{1}$
}

\begin{abstract}
RESUMO
Visou-se, com o presente estudo, avaliar os efeitos de seis anos de adubação orgânica sobre as características químicas de um Neossolo Regolítico cultivado com batata. Os tratamentos de adubação orgânica foram: 1) esterco (15 tha-1); 2) cultivo e incorporação da Crotalaria juncea L.; 3) combinação de metade da dose de esterco $\left(7,5 \mathrm{t} \mathrm{ha}^{-1}\right)$ com o cultivo e incorporação da Crotalaria juncea L. e 4) testemunha, sem fertilização. Os ensaios de adubação orgânica e cultivo da batata foram realizados no período de 1996 a 2003, com exceção de 1998 e 1999, devido à escassez de chuvas. Ao final de 2003 amostras de solo coletadas nas camadas de 0-20 e 20-40 cm de profundidade foram analisadas. A aplicação anual de esterco, combinado ou não com a crotalária, elevou os teores de $\mathrm{CO}, \mathrm{N}$ e $\mathrm{P}$ totais, e Zn na camada de 0-20 cm de profundidade, e os teores de P e K extraíveis, Mg e B nas duas camadas avaliadas. O cultivo e a incorporação anual da crotalária não exerceram efeito significativo nos teores de nutrientes do solo. Os resultados sugerem que a utilização de doses menores de esterco, combinadas com a incorporação de adubos verdes pode garantir a manutenção da fertilidade do solo em longo prazo.
\end{abstract}

Palavras-chave: esterco, Crotalaria juncea L., adubação verde, região semi-árida, micronutrientes

\section{Soil fertility changes in an Entisol following six years of application of organic manure}

\begin{abstract}
The objective of this study was to evaluate the effects of application of six years of organic manure with animal and/or cover crop on the fertility of an Entisol. Treatments were: 1$)$ animal manure $\left.\left(15 \mathrm{t} \mathrm{ha}^{-1}\right) ; 2\right)$ use of Crotalaria juncea L. as a cover crop; 3$)$ fertilization with half $\left(7.5 \mathrm{t} \mathrm{ha}^{-1}\right)$ of the usual doses of animal manure combined with C. juncea L. as a cover crop; and 4) control plots without application. The study was conducted from 1996 to 2003, but no fertilization was done in 1998 and 1999, due to severe droughts. At the end of 2003, soil samples from two depths (0-20 and 20-40 cm) were collected and analyzed to determine the nutrient concentrations. The annual fertilization with animal manure, with or without $\mathrm{C}$. juncea, increased soil extractable $\mathrm{P}, \mathrm{K}, \mathrm{Mg}$ and $\mathrm{B}$ in both soil layers, but increased soil organic carbon, total $\mathrm{N}$, total $\mathrm{P}$, and $\mathrm{Zn}$ only in the $0-20 \mathrm{~cm}$ layer. The cultivation and incorporation of $C$. juncea had no consistent effect on soil fertility. The results have shown that the application of lower doses of manure combined with $\mathrm{C}$. juncea maintained soil fertility at levels comparable to application of $15 \mathrm{tha}^{-1}$ of animal manure.
\end{abstract}

Key words: manure, Crotalaria juncea L., green manure, semi-arid region of NE Brazil, micronutrients 


\section{INTRODUÇÃO}

Em geral, os solos da região semi-árida do Nordeste do Brasil são pouco férteis devido principalmente, à baixa disponibilidade de N e P (Sampaio et al., 1995); a região é caracterizada pela predominância de pequenas propriedades com mão-de-obra familiar, cultivos agrícolas de subsistência e pecuária baseada no pastoreio da vegetação nativa. Os fertilizantes químicos comerciais são pouco utilizados em virtude do seu alto custo, do baixo poder aquisitivo da maioria dos agricultores e do risco proporcionado pela variabilidade do regime de chuvas (Nascimento et al., 2003); por este motivo, a fertilidade dos solos depende, sobretudo, do manejo da matéria orgânica do solo (Tiessen et al., 1994; Miyazawa et al., 2000; Nascimento et al., 2003; Salcedo, 2004).

O Neossolo Regolítico é um tipo de solo que apresenta textura arenosa e baixa capacidade de adsorção de nutrientes, quando comparado com solos argilosos (Tito et al., 1997); naturalmente, possui teor baixo de matéria orgânica e nitrogênio que diminuem, após alguns anos de uso (Jacomine, 1996). Apesar dessas limitações, na microrregião de Esperança, no Estado da Paraíba, este solo é intensamente utilizado na agricultura recebendo, anualmente, grandes quantidades de matéria orgânica (Tito et al., 1997).

O esterco animal é o principal adubo orgânico utilizado para a melhoria da fertilidade dos solos do semi-árido nordestino, mas a quantidade de esterco disponível nas propriedades é, em geral, insuficiente para suprir a demanda das culturas agrícolas (Holanda, 1990; Silva et al., 2007).

Nas áreas de maior tradição agrícola, como o agreste paraibano, vários agricultores compram esterco para a fertilização do solo em cultivos agrícolas de maior rentabilidade, como a batata, mas isto aumenta os custos de produção; outrossim, o esterco de baixa qualidade e/ou não-curtido, pode provocar a imobilização de nutrientes do solo nas semanas seguintes após sua incorporação, com limitações no crescimento e produtividade das espécies vegetais (Holanda, 1990; Palm et al., 2001; Silva, 2004). Alguns estudos na região Agreste da Paraíba demonstraram que o cultivo de cobertura e adubação verde com a crotalária (Crotalaria juncea L.) pode diminuir a necessidade de esterco e evitar a imobilização de nitrogênio do solo (Menezes et al., 2002; Silva, 2004); este efeito benéfico da crotalária decorre, sobremaneira, da capacidade dessa leguminosa de fixar $\mathrm{N}_{2}$ atmosférico em associação com rizóbios, porém a adubação verde com cultivos anuais de cobertura em longo prazo não é capaz de manter a fertilidade do solo, haja vista que os nutrientes retirados do solo pelo adubo verde são absorvidos pelas culturas agrícolas e exportados com a colheita. Com exceção do $\mathrm{N}$, é imprescindível uma fonte externa para repor os nutrientes retirados do solo.

Silva (2004) demonstrou que a incorporação de esterco, mesmo em doses menores que as usualmente empregadas, combinadas com o cultivo de leguminosas para adubação verde, é uma prática de manejo viável, pois além de evitar a imobilização de nitrogênio do solo e disponibilizar esse nu- triente de forma mais sincronizada com a demanda das culturas agrícolas é, também, capaz de manter a fertilidade do solo em longo prazo; entretanto, ainda são escassos os dados sobre o efeito da adubação orgânica em solos da região semi-árida.

Ante esse contexto objetivou-se, com o presente trabalho, avaliar os efeitos de seis anos de adubação orgânica com esterco, com Crotalaria juncea L. ou com esses dois adubos orgânicos combinados, sobre a fertilidade de um Neossolo Regolítico no Agreste da Paraíba.

\section{MATERIAL E MÉTODOS}

\section{Descrição da área do estudo}

A área do estudo localiza-se no Centro Agroecológico de São Miguel, município de Esperança, Agreste da Paraíba. A pluviosidade média anual é de 800 mm, com regime de chuvas unimodal, estação úmida de março a agosto e estação seca de setembro a fevereiro (Sabourin et al., 2000). O solo na área experimental está classificado como Neossolo Regolítico, com textura areia franca e declividade em torno de $0,05 \mathrm{~m} \mathrm{~m}^{-1}$.

Os tratamentos aplicados no experimento de adubação orgânica e cultivo da batata (Solanum tuberosum L.) consistiram em: plantio e incorporação da crotalária na época da floração (C); adição de $15 \mathrm{t} \mathrm{ha}^{-1} \mathrm{ano}^{-1}$ de esterco (E); plantio e incorporação da crotalária $+7,5$ t ha $^{-1}$ ano $^{-1}$ de esterco (CE) e testemunha sem esterco ou crotalária (T). As parcelas experimentais tinham área de $60 \mathrm{~m}^{2}$ (6 x $\left.10 \mathrm{~m}\right)$ e o delineamento experimental utilizado foi em blocos inteiramente casualizados com quatro repetições. O experimento foi repetido nos anos de 1996, 1997, 2000, 2001, 2002 e 2003, sempre nas mesmas parcelas experimentais (1998 e 1999 foram anos de seca e não houve plantio nem adubação do solo), de modo a verificar o efeito acumulativo dos tratamentos ao longo do tempo.

Maiores detalhes sobre o histórico do manejo do solo, a produtividade da batata e a dinâmica dos nutrientes do solo ao longo do período de 6 anos em que foi conduzido o estudo, são descritos em Silva (2004), Silva et al. (2007) e Silva \& Menezes (2007).

\section{Amostragem do solo}

Antes do início do experimento, em 1996, amostras de solo foram coletadas na profundidade de $0-20 \mathrm{~cm}$, ao acaso, dentro da área de cada um dos blocos da área experimental. As amostras foram secadas ao ar, tamisadas em peneiras de $2 \mathrm{~mm}$ de abertura, homogeneizadas e preparadas para a determinação das características químicas iniciais do solo da área experimental (Tabela 1). Essas amostras serviram como referência da fertilidade do solo na área experimental antes do início dos tratamentos de adubação orgânica. Ao final do período do estudo, em 2003, coletaram-se amostras de solo nas profundidades de 0-20 e 20-40 cm das parcelas experimentais, para verificar o impacto causado pelos adubos na fertilidade do solo nessas duas profundidades durante os seis anos de adubação orgânica. Os impactos dos tratamentos de 
adubação orgânica foram avaliados principalmente comparando-se a fertilidade do solo nas parcelas adubadas com o solo das parcelas controle que não receberam adubação.

Tabela 1. Atributos químicos e físicos do Neossolo Regolítico $(0-20 \mathrm{~cm})$ na área experimental em Esperança, PB, em 1996, antes do início da aplicação dos tratamentos de adubação orgânica

\begin{tabular}{|c|c|c|}
\hline Características do solo & Média $(n=4)$ & CV (\%) \\
\hline $\mathrm{pH}\left(\mathrm{H}_{2} \mathrm{O}\right)$ & 6,1 & 5,1 \\
\hline Carbono orgânico $\left(\mathrm{g} \mathrm{kg}^{-1}\right)$ & 6,0 & 15,2 \\
\hline $\mathrm{N}$-total $\left(\mathrm{g} \mathrm{kg}^{-1}\right)$ & 0,55 & 14,1 \\
\hline P-Mehlich-1 (mg kg-1) & 6,3 & 33,0 \\
\hline $\mathrm{S}\left(\mathrm{mg} \mathrm{kg}^{-1}\right)$ & 5,8 & 3,8 \\
\hline $\mathrm{K}\left(\mathrm{cmol}_{\mathrm{c}} \mathrm{kg}^{-1}\right)$ & 0,22 & 31,7 \\
\hline $\mathrm{Ca}\left(\mathrm{cmol}_{\mathrm{c}} \mathrm{kg}^{-1}\right)$ & 2,1 & 15,1 \\
\hline $\mathrm{Mg}\left(\mathrm{cmol}_{\mathrm{c}} \mathrm{kg}^{-1}\right)$ & 0,60 & 23,6 \\
\hline $\mathrm{Na}\left(\mathrm{cmol}_{\mathrm{c}} \mathrm{kg}^{-1}\right)$ & 0,09 & 27,0 \\
\hline $\mathrm{Al}\left(\mathrm{cmol}_{\mathrm{c}} \mathrm{kg}^{-1}\right)$ & 0,08 & 190,0 \\
\hline $\mathrm{Zn}\left(\mathrm{mg} \mathrm{kg}^{-1}\right)$ & 2,2 & 29,7 \\
\hline $\mathrm{Fe}\left(\mathrm{mg} \mathrm{kg}^{-1}\right)$ & 86 & 45,3 \\
\hline $\operatorname{Mn}\left(\mathrm{mg} \mathrm{kg}^{-1}\right)$ & 3,7 & 12,3 \\
\hline $\mathrm{Cu}\left(\mathrm{mg} \mathrm{kg}^{-1}\right)$ & 0,23 & 18,3 \\
\hline$B\left(\mathrm{mg} \mathrm{kg}^{-1}\right)$ & 0,61 & 19,9 \\
\hline Areia $\left(\right.$ dag $\left.\mathrm{kg}^{-1}\right)$ & 83 & 8,7 \\
\hline Silte $\left(\right.$ dag $\left.\mathrm{kg}^{-1}\right)$ & 11 & 6,3 \\
\hline Argila $\left(\right.$ dag $\left.\mathrm{kg}^{-1}\right)$ & 6 & 12,4 \\
\hline
\end{tabular}

$\mathrm{CV}$ - Coeficiente de variação

As amostras de solo foram analisadas quanto aos teores de C orgânico (Snyder \& Trofymow, 1984), N total, P total, P orgânico (Walker \& Adams, 1958), P extraível, $\mathrm{N}_{-} \mathrm{NH}_{4}{ }^{+}$, $\mathrm{N}-\mathrm{NO}_{3}{ }^{-}, \mathrm{K}^{+}, \mathrm{Ca}^{2+}, \mathrm{Mg}^{2+}, \mathrm{Na}^{+}$e $\mathrm{Al}^{3+}, \mathrm{S}_{-} \mathrm{SO}_{4}{ }^{2-}, \mathrm{Zn}, \mathrm{Fe}, \mathrm{Mn}, \mathrm{Cu}$, $\mathrm{B}$ e valores de $\mathrm{pH}$ em água (1:2,5 v/v) (EMBRAPA, 1997).

\section{Análise estatística}

As características químicas do solo nas camadas avaliadas foram submetidas a análise de variância e as médias comparadas através do teste Tukey a 5\% de probabilidade, com o uso do programa estatístico SISVAR (Ferreira, 2003).

\section{RESULTADOS E DISCUSSÃO}

Em 1996, antes do início da aplicação dos adubos orgânicos, o solo da área experimental apresentava baixos teores de $\mathrm{C}$ orgânico, $\mathrm{N}$ total, $\mathrm{P}$ extraível e alumínio, teores médios de K, Ca e Mg trocáveis, pH fracamente ácido e textura areia franca (Tabela 1), características típicas da maioria dos Neossolos Regolíticos da região semi-árida nordestina (Menezes et al., 2005). Valores superiores ao teor de P-extraível (Mehlich-1) para solos da região semi-árida, foram encontrados por Silveira et al. (2006), que determinaram as concentrações de $\mathrm{P}$ nas ordens de solos mais comuns no semiárido de Pernambuco e Paraíba, observando teores médios de 14,6 e $6,7 \mathrm{mg} \mathrm{kg}^{-1}$ de $\mathrm{P}$ nas camadas de $0-20$ e $20-40 \mathrm{~cm}$, respectivamente.

Quanto aos níveis de $\mathrm{S}$ e micronutrientes em solos do semi-árido, são poucos os dados disponíveis na literatura para efeito de comparação com os resultados do presente estudo; entre esses dados, estão os relatados por Oliveira \& Nascimento (2006) e Nascimento et al. (2006), que determinaram os níveis de micronutrientes nos solos de referência do estado de Pernambuco e observaram, no Neossolo Regolítico, valores que variaram entre 0,9 e 79,7 para o $\mathrm{Mn}, 20,9$ e 36,1 para o $\mathrm{Fe}, 6,7$ e 7,0 para o $\mathrm{Cu}$ e 1,8 e $3,6 \mathrm{mg} \mathrm{dm}^{-3}$ para o Zn. Em comparação com esses valores, o solo do presente estudo apresentava, em 1996, teores semelhantes de Zn e Mn, baixos de $\mathrm{Cu}$ e altos de $\mathrm{Fe}$.

\section{Efeitos da adubação orgânica sobre o C, N, P e S do solo}

A aplicação de esterco nos tratamentos E e CE elevou significativamente os teores de carbono orgânico (CO) na camada de 0-20 cm de profundidade, em relação ao tratamento testemunha, mas o cultivo e a incorporação da crotalária (C) não exerceram efeitos significativos sobre o CO do solo (Tabela 2); nenhum dos tratamentos elevou significativamente o CO do solo na camada de $20-40 \mathrm{~cm}$ de profundidade. A ausência de efeitos significativos em camadas sub-superficiais do solo foi também observada em outros trabalhos com aplicação de adubos orgânicos (Alcântara, 1998; Nascimento et al., 2003; Silva et al., 2004).

Tabela 2. Teores médios ${ }^{1}$ de carbono orgânico (CO), nitrogênio total $(\mathrm{Nt})$, nitrogênio inorgânico (Ni), fósforo total (Pt), fósforo orgânico $(\mathrm{Po})$, e fósforo extraível (Pm) e enxofre disponível (S) em duas profundidades de um Neossolo Regolítico após seis anos de adubação orgânica com esterco e/ou crotalária

\begin{tabular}{|c|c|c|c|c|c|c|c|}
\hline \multirow{2}{*}{ Tratamentos ${ }^{2}$} & $\mathrm{CO}$ & $\mathrm{Nt}$ & $\mathrm{Ni}$ & $\mathrm{Pt}$ & Po & $\mathrm{Pm}$ & $S$ \\
\hline & \multicolumn{2}{|c|}{$\left(g^{k g}{ }^{-1}\right)$} & \multicolumn{5}{|c|}{$\left(\mathrm{mg} \mathrm{kg}^{-1}\right)$} \\
\hline & \multicolumn{7}{|c|}{$0-20 \mathrm{~cm}$} \\
\hline $\mathrm{T}$ & $4,5 \mathrm{c}$ & $0,48 \mathrm{~b}$ & $6,1 \mathrm{~b}$ & $126,3 \mathrm{c}$ & $39,7 \mathrm{c}$ & $10,9 \mathrm{~b}$ & $65,2 \mathrm{a}$ \\
\hline $\mathrm{CE}$ & $6,5 a b$ & $0,77 \mathrm{a}$ & $16,7 \mathrm{a}$ & $203,6 a b$ & $63,0 a b$ & $37,0 \mathrm{a}$ & $63,0 \mathrm{a}$ \\
\hline$E$ & $7,3 \mathrm{a}$ & $0,80 \mathrm{a}$ & $13,4 \mathrm{a}$ & $236,5 \mathrm{a}$ & $72,1 \mathrm{a}$ & $46,2 \mathrm{a}$ & $63,2 \mathrm{a}$ \\
\hline C & $5,3 \mathrm{bc}$ & $0,54 \mathrm{~b}$ & $14,8 \mathrm{a}$ & $142,8 \mathrm{bc}$ & 50,2 bc & $11,5 \mathrm{~b}$ & $66,3 \mathrm{a}$ \\
\hline Média & 5,9 & 0,65 & 12,8 & 144,6 & 56,3 & 26,4 & 64,4 \\
\hline \multirow[t]{2}{*}{ C.V. $(\%)^{3}$} & 14,5 & 12,7 & 31,5 & 20,1 & 17,4 & 18,2 & 4,8 \\
\hline & \multicolumn{7}{|c|}{$20-40 \mathrm{~cm}$} \\
\hline $\mathrm{T}$ & $4,5 \mathrm{a}$ & $0,42 \mathrm{~b}$ & $8,3 \mathrm{~b}$ & 72,2 c & $30,0 \mathrm{~b}$ & $9,4 \mathrm{~b}$ & $63,0 \mathrm{a}$ \\
\hline CE & $5,5 \mathrm{a}$ & $0,61 \mathrm{a}$ & $17,0 \mathrm{a}$ & $116,2 \mathrm{ab}$ & $41,4 a b$ & $22,8 \mathrm{a}$ & $60,9 \mathrm{a}$ \\
\hline $\mathrm{E}$ & $5,4 \mathrm{a}$ & $0,56 a b$ & $12,3 a b$ & $131,4 \mathrm{a}$ & $43,5 \mathrm{a}$ & $30,4 \mathrm{a}$ & $59,4 \mathrm{a}$ \\
\hline C & $4,3 \mathrm{a}$ & $0,49 a b$ & $13,0 a b$ & 85,0 bc & $39,3 a b$ & $7,5 \mathrm{~b}$ & $58,5 \mathrm{a}$ \\
\hline Média & 4,9 & 0,52 & 12,7 & 101,2 & 38,6 & 17,6 & 60,5 \\
\hline CV $(\%)^{3}$ & 14,0 & 15,5 & 20,2 & 17,3 & 15,2 & 23,8 & 3,8 \\
\hline
\end{tabular}

${ }^{1}$ Médias na coluna seguidas da mesma letra dentro de cada profundidade não diferem entre si pelo teste Tukey a $5 \%$ de probabilidade

${ }^{2} \mathrm{~T}$ - testemunha; CE - plantio e incorporação da crotalária $+7,5$ tha-1 de esterco; $\mathrm{E}-15 \mathrm{tha}^{-1}$ de esterco; C - plantio e incorporação da crotalária

${ }^{3} \mathrm{CV}$ - coeficiente de variação

De forma semelhante, a aplicação de esterco nos tratamentos E e CE aumentou a concentração de $\mathrm{N}$ total $(\mathrm{Nt})$ na camada de $0-20 \mathrm{~cm}$ do solo em 142 e 147\%, respectivamente (Tabela 2); entretanto, em contraste ao previsto, o plantio e a incorporação da crotalária por longo período não alteraram significativamente o teor de Nt do solo. Na camada de 20-40 cm, os teores de Nt e nitrogênio inorgânico (Ni) foram maiores apenas no tratamento $\mathrm{CE}$ em relação ao tratamento T (Tabela 2); além disso, em apenas 6 anos de aplicações anuais, a adubação orgânica com 15 t ha-1 de esterco 
(tratamento E) aumentou significativamente o teor de $\mathrm{P}$ total do solo em 187 e 165\%, comparado aos tratamentos C e T, respectivamente; o teor de fósforo orgânico (Po) foi maior para o tratamento $\mathrm{E}$, que diferiu dos tratamentos $\mathrm{C}$ e $\mathrm{T}$; simultaneamente, o teor de fósforo extraído pelo extrator Mehlich-1 (Pm) na camada de 0-20 cm aumentou 587 e 733\% no solo dos tratamentos CE e E, respectivamente (Tabela 2), enfatizando a importância da aplicação de esterco como fornecedor de P para as culturas agrícolas. Uma vez que nos tratamentos C e T não se aplicou esterco, uma possível explicação para o aumento do teor de $\mathrm{P}$ nesse solo ao longo do período de cultivo pode ser esclarecida pela ação das plantas espontâneas e da crotalária, que podem ter contribuído para disponibilizar o fósforo que se encontrava no solo em formas menos lábeis. Alguns estudos já relataram que as leguminosas podem ter papel significativo na disponibilização para o solo de $\mathrm{P}$ que se encontra em formas menos lábeis (Novais \& Smyth, 1999).

Em relação ao enxofre não se observaram diferenças significativas entre os tratamentos avaliados em ambas as camadas de solo amostradas, mas os teores de S foram superiores aos verificados na literatura (Alcântara, 1998; Furtini Neto et al., 2001; Rein \& Sousa, 2004). Ressalta-se que os teores de carbono orgânico e enxofre do solo foram aumentados significativamente durante o período de incorporação dos adubos orgânicos (Tabela 2), fato coerente, já que a matéria orgânica também é fonte de enxofre no solo (Malavolta, 1980; Stevenson, 1994; Paul \& Clark, 1996; Moreira \& Siqueira, 2002); sendo assim, observa-se que a adição de matéria orgânica ao solo no presente estudo foi capaz não só de garantir a manutenção de níveis adequados de enxofre no solo como, também, elevar significativamente esses níveis, após um período de seis anos.

De maneira geral, os Neossolos Regolíticos da região Agreste da Paraíba são solos arenosos e de baixa fertilidade, normalmente com baixa disponibilidade de N e P, como a maioria dos solos da região semi-árida (Sampaio et al., 1995; Menezes et al., 2005), motivo pelo qual os aumentos nos níveis de carbono e nitrogênio orgânicos e de fósforo do solo podem apresentar forte impacto positivo sobre a produtividade agrícola, porém é importante destacar que, apesar do tratamento E ter proporcionado maiores aumentos nas concentrações de $\mathrm{C}$, $\mathrm{N}$ e $\mathrm{P}$ do solo ao final do período de 6 anos do estudo, as maiores produtividades de batata, ao longo desse período, foram obtidas no tratamento $\mathrm{CE}$, devido à melhor sincronização entre a demanda de $\mathrm{N}$ pela batata e a disponibilidade desse elemento no solo, ao longo dos ciclos de cultivo a cada ano (Silva, 2004); em resumo, quando da escolha de um adubo orgânico deve-se levar em conta não apenas a quantidade de nutrientes adicionada mas, também, a qualidade do adubo e sua capacidade de disponibilizar os nutrientes para os cultivos agrícolas no momento adequado (Vanlauwe et al., 2005).

\section{Efeitos sobre o pH e as bases trocáveis do solo}

A aplicação de $15 \mathrm{t} \mathrm{ha}^{-1}$ de esterco aumentou significativamente o pH do solo em relação ao tratamento apenas com o plantio e incorporação da crotalária (C), tornando o solo fracamente alcalino nas duas camadas avaliadas (Tabela 3). No tratamento CE observou-se efeito semelhante, mas só houve aumento significativo do $\mathrm{pH}$ do solo na camada de $20-40 \mathrm{~cm}$.

Tabela 3. Teores médios ${ }^{1}$ de bases trocáveis, acidez trocável e pH em duas profundidades de um Neossolo Regolítico após seis anos de adubação orgânica com esterco e/ou crotalária

\begin{tabular}{|c|c|c|c|c|c|c|c|c|}
\hline \multirow{2}{*}{ Tratam. $^{2}$} & $\mathrm{Ca}$ & $\mathrm{Mg}$ & $\mathrm{K}$ & $\mathrm{Na}$ & $\mathrm{Al}+\mathrm{H}$ & $\mathrm{SB}^{3}$ & CTC $^{4}$ & \multirow{2}{*}{$\begin{array}{c}\mathrm{pH} \\
\left(\mathrm{H}_{2} \mathrm{O}\right)\end{array}$} \\
\hline & \multicolumn{7}{|c|}{$\left(\mathrm{cmol}_{\mathrm{c}} \mathrm{kg}^{-1}\right)$} & \\
\hline & \multicolumn{8}{|c|}{$0-20 \mathrm{~cm}$} \\
\hline $\mathrm{T}$ & $1,4 \mathrm{~b}$ & $0,50 \mathrm{~b}$ & $0,31 b$ & $0,05 b$ & $0,05 \mathrm{a}$ & $2,17 \mathrm{c}$ & $2,22 \mathrm{c}$ & $6,9 \mathrm{ab}$ \\
\hline CE & $1,8 a b$ & $1,04 a$ & $0,94 a$ & $0,32 \mathrm{a}$ & $0,05 a$ & $3,76 a b$ & $3,80 a b$ & $6,9 a b$ \\
\hline$E$ & 2,3a & $1,23 a$ & $0,95 a$ & $0,32 \mathrm{a}$ & $0,11 \mathrm{a}$ & $4,49 a$ & $4,61 \mathrm{a}$ & $7,5 \mathrm{a}$ \\
\hline C & $1,5 a b$ & $0,59 b$ & $0,38 \mathrm{~b}$ & $0,05 b$ & $0,10 \mathrm{a}$ & $2,46 \mathrm{bc}$ & $2,56 \mathrm{bc}$ & $6,4 \mathrm{~b}$ \\
\hline Média & 1,7 & 0,42 & 0,64 & 0,18 & 0,07 & 3,22 & 3,30 & 6,9 \\
\hline \multirow[t]{2}{*}{ C.V. $(\%)^{5}$} & 22,4 & 20,6 & 30,7 & 18,0 & 40,0 & 18,7 & 18,3 & 5,7 \\
\hline & \multicolumn{8}{|c|}{$20-40 \mathrm{~cm}$} \\
\hline $\mathrm{T}$ & $1,4 b$ & $0,54 b$ & $0,23 b$ & $0,05 b$ & $0,06 \mathrm{a}$ & $2,16 \mathrm{~b}$ & $2,21 b$ & $6,4 \mathrm{bc}$ \\
\hline $\mathrm{CE}$ & $1,9 a b$ & $0,99 a$ & $0,51 a$ & $0,17 \mathrm{a}$ & $0,06 \mathrm{a}$ & $3,43 a$ & $3,48 a$ & $7,1 \mathrm{ab}$ \\
\hline $\mathrm{E}$ & $2,2 \mathrm{a}$ & $1,02 \mathrm{a}$ & $0,58 \mathrm{a}$ & $0,16 \mathrm{a}$ & $0,06 \mathrm{a}$ & $3,76 \mathrm{a}$ & $3,81 a$ & $7,5 \mathrm{a}$ \\
\hline $\mathrm{C}$ & $1,5 a b$ & $0,57 b$ & $0,21 b$ & $0,05 b$ & $0,06 \mathrm{a}$ & $2,27 b$ & $2,32 b$ & $5,9 \mathrm{c}$ \\
\hline Média & 1,7 & 0,78 & 0,39 & 0,11 & 0,06 & 2,90 & 2,96 & 6,7 \\
\hline C.V. $(\%)^{5}$ & 18,8 & 15,2 & 28,8 & 15,4 & 0,0 & 17,1 & 16,7 & 5,0 \\
\hline
\end{tabular}

${ }^{1}$ Médias na coluna seguidas da mesma letra dentro de cada profundidade não diferem entre si pelo teste Tukey a $5 \%$ de probabilidade

${ }^{2} \mathrm{~T}$ - testemunha; CE - plantio e incorporação da crotalária $+7,5 \mathrm{tha}^{-1}$ de esterco; $\mathrm{E}-15 \mathrm{t} \mathrm{ha}^{-1}$ de esterco; C - plantio e incorporação da crotalária

${ }^{3}$ Soma de bases

${ }^{4}$ capacidade de troca catiônica efetiva

${ }^{5} \mathrm{CV}$ - coeficiente de variação

Constatou-se, nos tratamentos E e CE, elevação significativa nos teores de bases trocáveis do solo nas duas profundidades avaliadas (Tabela 3); de forma idêntica, houve aumento da CTC do solo nos tratamentos E e CE nas duas camadas do solo, provavelmente em decorrência do aumento do carbono orgânico do solo, em virtude da aplicação do esterco (Tabela 2); outros estudos reportaram comportamento semelhante nas características do solo após aumento do teor de matéria orgânica do solo (Stevenson, 1994; Stewart \& Robinson, 1997; Bayer et al. 2003). Em relação aos efeitos dos adubos verdes na fertilidade do solo, Barreto \& Fernandes (2001) também verificaram aumentos nas características químicas ( $\mathrm{Ca}+\mathrm{Mg}$ e $\mathrm{pH}$ ) em solos dos tabuleiros costeiros, após a incorporação de biomassa de gliricídia ou leucena. Essas mudanças nas características químicas após a aplicação de adubos verdes em solos tropicais, como o aumento do pH e imobilização do Al, são explicadas pela formação de complexos organo-metálicos (Miyazawa et al., 2000; Ceretta et al., 2003).

Os tratamentos E e CE apresentaram teores altos de Mg nas duas camadas avaliadas (Tabela 3), enquanto o solo dos tratamentos C e T mostrou teores médios, de acordo com Tomé Jr. (1997) e Furtini Neto et al. (2001). Na região do Agreste paraibano, teores maiores de Mg que os encontrados no Neossolo Regolítico foram constatados em um Luvissolo, quando cultivado durante 3 anos com diferentes leguminosas (Nascimento et al., 2003). Os tratamentos C e T indicaram teores adequados de $\mathrm{K}$, mas os tratamentos que 
receberam esterco (E e CE) indicaram teores muito altos, de acordo com Furtini Neto et al. (2001). Em solos pouco intemperizados, como os Neossolos Regolíticos, a liberação de K pelos minerais, como feldspatos, é capaz de suprir a demanda das plantas, durante alguns ciclos das culturas (Curi et al., 2005); desta forma, diz-se que, inicialmente, não é necessário corrigir o teor de potássio em solos como o do presente estudo; entretanto, em áreas cultivadas longos períodos é recomendável monitorar os teores desse nutriente no solo para evitar deficiências às plantas.

Com relação aos teores de $\mathrm{Al}^{3+}$ e apesar dos adubos orgânicos não terem causado efeitos significativos, vê-se que as concentrações no Neossolo após seis anos de aplicação dos adubos orgânicos são classificadas como muito baixas (Furtini Neto et al., 2001), ou seja, não prejudiciais para as culturas (Tomé Jr., 1997).

\section{Efeitos sobre os micronutrientes do solo}

As aplicações de adubos orgânicos ao longo do período do estudo influenciaram significativamente os teores de micronutrientes do solo (Kiehl, 1985). Entretanto, só foi possível avaliar comparativamente os efeitos da adubação ao longo do tempo para a camada de $0-20 \mathrm{~cm}$ do solo, uma vez que não há dados para a camada de 20-40 cm em 1996, quando iniciou-se a aplicação dos adubos. Verificaram-se, para o zinco $(\mathrm{Zn})$, maiores incrementos para os tratamentos E e CE, com aumentos de 151 e $88 \%$, respectivamente, em relação ao teor desse elemento na camada de 0-20 cm do solo, no ano de 1996 (Tabela 1). De acordo com Furtini Neto et al. (2001), os níveis de Zn em todos os tratamentos e profundidades avaliadas no presente estudo, foram considerados muito bons, tanto no início do estudo como após os seis anos de aplicação dos adubos (Tabela 1). Comportamento semelhante ao do $\mathrm{Zn}$ foi verificado para o ferro (Fe), que também apresentou teores muito bons. O manganês $(\mathrm{Mn})$ e o cobre $(\mathrm{Cu})$ também sofreram aumentos com a aplicação contínua dos adubos orgânicos (Tabela 4), esses dois com teores considerados adequados no solo. Os teores de boro (B) ao longo do período de aplicação dos adubos orgânicos e cultivo da batata, aumentaram nos tratamentos CE e E (Tabela 4), sendo considerados nível bom e muito bom, de acordo com Furtini Neto et al. (2001), correspondendo a um incremento de 34,4 e $65,6 \%$, respectivamente. De forma contrastante, os tratamentos $\mathrm{C}$ e $\mathrm{T}$ reduziram os teores de B no solo ao longo do período, em 60 e $72 \%$, respectivamente. Com exceção dos teores de B nos tratamentos C e T em ambas as camadas avaliadas, os demais teores dos diferentes micronutrientes avaliados enquadram a fertilidade desse solo como adequada (Vitti \& Malavolta, 1999). O que se pode recomendar para o elemento boro, é que o Neossolo Regolítico no Agreste paraibano necessita de uma adubação de complementação no período do plantio, quando não se utilizar a adubação com esterco. Alguns fatores podem ter contribuído para que o solo dos tratamentos C e T apresentassem baixos teores de B; como o plantio e a incorporação da crotalária (C) e a incorporação das ervas espontâneas ao solo $(\mathrm{T})$ provavelmente não são suficientes para repor o B que é exportado pelas cultu- ras no momento da colheita; além disso, o B pode ser lixiviado do solo principalmente daqueles de textura leve, como é o caso do solo do presente estudo (Raij, 1991); por outro lado e uma vez que o boro disponível pode ser retido pela matéria orgânica do solo, práticas de adubação orgânica poderiam solucionar a deficiência desse micronutriente (Furtini Neto et al., 2001) e, então, a incorporação de resíduos orgânicos, mesmo em pequenas quantidades em solos arenosos com baixo teor de matéria orgânica, pode ser uma prática de vital importância para a disponibilidade de micronutrientes.

Tabela 4. Teores médios ${ }^{1}$ de micronutrientes em duas profundidades de um Neossolo Regolítico após seis anos de adubação orgânica com esterco e/ou crotalária

\begin{tabular}{|c|c|c|c|c|c|}
\hline \multirow{2}{*}{ Tratamentos $^{2}$} & $\mathrm{Zn}$ & $\mathrm{Fe}$ & $\mathrm{Mn}$ & $\mathrm{Cu}$ & B \\
\hline & \multicolumn{5}{|c|}{$\left(\mathrm{mg} \mathrm{kg}^{-1}\right)$} \\
\hline & \multicolumn{5}{|c|}{$0-20 \mathrm{~cm}$} \\
\hline $\mathrm{T}$ & $2,6 \mathrm{c}$ & $89 a$ & $21,5 \mathrm{~b}$ & $1,94 \mathrm{a}$ & $0,17 \mathrm{~b}$ \\
\hline CE & $4,1 \mathrm{~b}$ & $124 \mathrm{a}$ & 29,6 b & $1,65 \mathrm{a}$ & $0,82 \mathrm{a}$ \\
\hline$E$ & $5,5 \mathrm{a}$ & $103 \mathrm{a}$ & $40,7 \mathrm{a}$ & $2,58 \mathrm{a}$ & $1,01 \mathrm{a}$ \\
\hline $\mathrm{C}$ & $2,5 \mathrm{c}$ & $112 \mathrm{a}$ & $25,3 \mathrm{~b}$ & $2,20 \mathrm{a}$ & $0,24 \mathrm{~b}$ \\
\hline Média & 3,7 & 107 & 29,3 & 2,09 & 0,56 \\
\hline \multirow[t]{2}{*}{$\mathrm{CV}(\%)^{3}$} & 9,8 & 24,1 & 13,0 & 24,1 & 19,6 \\
\hline & \multicolumn{5}{|c|}{$20-40 \mathrm{~cm}$} \\
\hline $\mathrm{T}$ & $2,3 a b$ & $116 \mathrm{~b}$ & 17,4 b & $1,36 \mathrm{a}$ & $0,24 \mathrm{~b}$ \\
\hline CE & $2,6 a b$ & $138 a b$ & $22,4 a b$ & $1,70 \mathrm{a}$ & $0,68 \mathrm{a}$ \\
\hline $\mathrm{E}$ & $3,3 \mathrm{a}$ & $172 a b$ & $31,2 \mathrm{a}$ & $2,47 \mathrm{a}$ & $0,74 \mathrm{a}$ \\
\hline C & $2,0 \mathrm{~b}$ & $181 \mathrm{a}$ & $23,8 \mathrm{ab}$ & $2,40 \mathrm{a}$ & $0,32 \mathrm{~b}$ \\
\hline Média & 2,6 & 151,9 & 23,7 & 1,98 & 0,49 \\
\hline CV $(\%)^{3}$ & 19,3 & 19,1 & 20,2 & 41,4 & 21,9 \\
\hline
\end{tabular}

${ }_{1}^{1}$ Médias na coluna seguidas da mesma letra dentro de cada profundidade não diferem entre si pelo teste Tukey a $5 \%$ de probabilidade

${ }^{2} \mathrm{~T}$ - testemunha; CE - plantio e incorporação da crotalária $+7,5$ tha $^{-1}$ de esterco; $\mathrm{E}-15$ t ha- $^{-1}$ de esterco; C - plantio e incorporação da crotalária

${ }^{3} \mathrm{CV}$ - coeficiente de variação

\section{CONCLUSÕES}

1. A adubação orgânica com $15 \mathrm{tha}^{-1}$ de esterco, aumentou significativamente os teores de carbono, nutrientes, $\mathrm{pH}$, e a capacidade de troca catiônica do solo, nas camadas de 0-20 e 20-40 cm de profundidade após um período de seis anos de adubação, em relação às parcelas testemunha que não receberam adubação.

2. A adubação com apenas $7,5 \mathrm{t} \mathrm{ha}^{-1}$ de esterco, metade da dose usualmente empregada pelos agricultores, quando combinada com a adubação verde com crotalária, mostrou efeito semelhante à aplicação de $15 \mathrm{t} \mathrm{ha}^{-1}$ de esterco sobre a fertilidade do solo, ao final dos seis anos de adubação.

3. A adubação verde com crotalária não mostrou efeito positivo sobre a fertilidade do solo.

\section{AGRADECIMENTOS}

Ao CNPq, CAPES e Inter American Institute for Global Change Research (IAI - CRN001) pelo apoio financeiro com vistas à realização do presente trabalho. 


\section{LITERATURA CITADA}

Alcântara, F. A. Adubação verde na recuperação da fertilidade de um solo degradado. Lavras: UFLA, 1998. 104p. Dissertação Mestrado

Barreto, A. C.; Fernandes, M. F. Cultivo de Gliricídia sepium e Leucaena leucocephala em alamedas visando a melhoria dos solos dos tabuleiros costeiros. Pesquisa Agropecuária Brasileira, v.36, n.10, p.1287-1293, 2001.

Bayer, C.; Spagnollo, E.; Wildner, L. P.; Ernani, P. R.; Albuquerque, J. A. Incremento de carbono e nitrogênio num latossolo pelo uso de plantas estivais para cobertura do solo. Ciência Rural, v.33, n.3, p.469 - 475, 2003.

Ceretta, C. A.; Durigon, R.; Basso, C. J.; Barcellos, L. A. R.; Vieira, F. C. B. Características químicas de solo sob aplicação de esterco líquido de suínos em pastagem natural. Pesquisa Agropecuária Brasileira, v.38, n.6, p.729-735, 2003.

Curi, N.; Kampf, N.; Marques, J. J. Mineralogia e formas de potássio em solos brasileiros. In: Yamada, T.; Roberts, T. L. (ed). Potássio na agricultura brasileira. Piracicaba: Potafos, 2005. p.71-91.

EMBRAPA - Empresa Brasileira de Pesquisa Agropecuária. Serviço Nacional de Levantamento e Conservação de Solos. Manual de métodos de análise de solos. 2.ed. Rio de Janeiro: Embrapa, 1997. 212p.

Ferreira, D. F. SISVAR software: Versão 4.6. Lavras: DEX/UFLA, 2003. Software.

Furtini Neto, A. E.; Vale, F. R.; Resende, A. V.; Guilherme, L. R. G.; Guedes, G. A. A. Fertilidade do solo. Lavras: UFLA/FAEPE, 2001. 252p.

Holanda, J. S. Esterco de curral: Composição, preservação e adubação. Natal: EMPARN, 1990. 69p. Documento, 17.

Jacomine, P. K. T. Solos sob caatingas - características e uso agrícola. In: Alvarez V., V. H.; Fontes, L. E. F.; Fontes, M. P. F. (ed). O solo nos grandes domínios morfoclimáticos do Brasil e o desenvolvimento sustentado. Viçosa: SBCS; UFV, DPS, 1996. p.95-111.

Kiehl, E. J. Fertilizantes orgânicos. Piracicaba: Agronômica Ceres, 1985. 492p.

Malavolta, E. Elementos de nutrição mineral de plantas. São Paulo: Agronômica Ceres, 1980. 251p.

Menezes, R. S. C.; Garrido, M. S; Marin, A. M. P. Fertilidade dos solos no semi-árido. In: Congresso Brasileiro de Ciência do Solo, 30, 2005, Recife. Anais... Recife: UFRPE, 2005. CD Rom.

Menezes, R. S. C.; Sampaio, E. V. S. B.; Silveira, L. M.; Tiessen, H.; Salcedo, I. H. Produção de batatinha com incorporação de esterco e/ou crotalária no Agreste paraibano. In: Silveira, L.; Petersen, P.; Sabourin, E. (org). Agricultura familiar e agroecologia no Semi-Árido: Avanços a partir do agreste da Paraíba. Rio de Janeiro: AS-PTA, 2002, p.261-270.

Miyazawa, M.; Pavan, M. A.; Franchini, J. C. Neutralização da acidez do perfil do solo por resíduos vegetais. Piracicaba: Potafos, Informações Agronômicas. 2000. 8p. Encarte Técnico, 92.

Moreira, F. M. S.; Siqueira, J. O. Microbiologia e bioquímica do solo. Lavras: UFLA, 2002. 626p.
Nascimento, C. W. A.; Oliveira, A. B.; Ribeiro, M. R.; Melo, E. E. C. Distribuition and availability of zinc and copper in Benchmark soils of Pernambuco State, Brazil. Communications Soil Science Plant Analysis, v.37, p.109-125, 2006.

Nascimento, J. T.; Silva, I. F.; Santiago, R. D.; Silva Neto, L. F. Efeito de leguminosas nas características químicas e matéria orgânica de um solo degradado. Revista Brasileira de Engenharia Agrícola e Ambiental, v.7, n.3. p.457-462, 2003.

Novais, R. F.; Smyth, T. J. Fósforo em solo e planta em condições tropicais. Viçosa: UFV/DPS, 1999. 399p.

Oliveira, A. B.; Nascimento, C. W. A. Formas de manganês e ferro em solos de referência de Pernambuco. Revista Brasileira de Ciência do Solo, v.30, n.1 p.99-110, 2006.

Palm, C. A; Giller, K. E.; Mafongoya, P. L.; Swift, M. J. Management of organic matter in the tropics: translating theory into practice. Nutrient Cycling in Agroecosystems, v.61, p.63-75, 2001.

Paul, E. A.; Clark, F. E. Soil microbiology and biochemistry. 2.ed. New York: Academic Press, 1996. 340p.

Raij, B. van. Fertilidade do solo e adubação. São Paulo: Ceres, Potafos, 1991. 343p.

Rein, T. A.; Sousa, D. M. G. Adubação com enxofre. In: Sousa, D. M. G.; Lobato, E. (ed). Cerrado: correção do solo e adubação. 2.ed. Brasília: Embrapa Informação Tecnológica, 2004. p.185-226.

Sabourin, E.; Silveira, L. M.; Tonneau, J. P.; Sidersky, P. Fertilidade e agricultura familiar no Agreste paraibano: Um estudo sobre o manejo da biomassa. Esperança: CIRAD-TERA/ASPTA, 2000. 59p.

Salcedo, I. H. Fertilidade do solo e agricultura de subsistência: Desafios para o Semi-árido Nordestino. In: Fertibio, 2004, Lages. Anais... Lages: UDESC, 2004. CD-Rom.

Sampaio, E. V. S. B.; Salcedo, I. H.; Silva, V. M.; Alves, G. D. Capacidade de suprimento de $\mathrm{N}$ e resposta à fertilização de 20 solos de Pernambuco. Revista Brasileira de Ciência do Solo, v.20, n.1, p.269-279. 1995.

Silva, J.; Silva, P. S. L.; Oliveira, M.; Silva, K. M. B. Efeito de esterco bovino sobre os rendimentos de espigas verdes e de grãos de milho. Horticultura Brasileira, v.22, n.2, p.326-331, 2004.

Silva, T. O. Adubação orgânica da batata (Solanum tuberosum L.) com esterco e/ou Crotalaria juncea em um Neossolo Regolítico. Recife: UFPE, 2004. 48p. Dissertação Mestrado

Silva, T. O.; Menezes, R. S. C. Adubação orgânica da batata com esterco e, ou, Crotalaria juncea. II. Disponibilidade de N, P e K no solo ao longo do ciclo de cultivo. Revista Brasileira de Ciência do Solo, v.31, n.1, p.51-61, 2007.

Silva, T. O.; Menezes, R. S. C.; Tiessen, H.; Sampaio, E. V. S. B.; Salcedo, I. H.; Silveira, L. M. Adubação orgânica da batata com esterco e, ou, Crotalaria juncea. I. Produtividade vegetal e estoque de nutrientes no solo em longo prazo. Revista Brasileira de Ciência do Solo, v.31, n.1 p.39-49, 2007.

Silveira, M. M. L.; Araújo, M. S. B.; Sampaio, E. V. S.B. Distribuição de fósforo em diferentes ordens de solo do Semi-Árido da Paraíba e de Pernambuco. Revista Brasileira de Ciência do Solo, v.30, n.2 p.281-291, 2006.

Snyder, J. D.; Trofymow, J. A. A rapid accurate wet oxidation diffusion procedure for determining organic and inorganic carbon in plant and soil samples. Communications in Soil Science Plant Analysis, v.15, n.5, p.587-597, 1984. 
Stevenson, F. J. Húmus chemistry: gênesis, composition, reactions. New York: John Wiley \& Sons, 1994. 486p.

Stewart, B. A.; Robinson, C. A. Are agroecosystems sustainable in semiarid regions? Advances in Agronomy, v.60. p.191-228. 1997.

Tiessen, H.; Cuevas, E. Chacon, P. The role of soil organic matter in sustaining soil fertility, Nature, v.371, p.783785, 1994.

Tito, G. A.; Chaves, L. E. G.; Carvalho, H. O.; Azevedo, N. C. Aplicação de bentonita em um regossolo eutrófico. II. Efeitos sobre as propriedades químicas do solo. Revista Brasileira de Engenharia Agrícola e Ambiental, v.1, n.1, p.25-27, 1997.

Tomé Júnior, J. B. Manual para interpretação de análise de solo. Guaíba: Agropecuária, 1997. 247p.
Vanlauwe, B.; Gachengo, C.; Sheperd, K.; Barrios, E.; Palm, C. A. Laboratory validation of a resource quality-based conceptual framework for organic matter management. Soil Science Society America Journal, v.69, p.1135-1145, 2005.

Vitti, G. C.; Malavolta, E. Atingir o patamar de produtividade alcançado com o uso de fertilizantes minerais via adubação orgânica: uma expectativa irreal? In: Siqueira, J. O.; Moreira, F. M. S.; Lopes, A. S; Guilherme, L. R. G.; Faquin, V.; Furtini Neto, A. E.; Carvalho, J.G. (ed.). Inter-relação fertilidade, biologia do solo e nutrição de plantas. Lavras: SBCS/UFLA/DCS, 1999. p.163-169.

Walker, T. M.; Adams, A. F. R. Studies on soil organic matter. I. Influence of phosphorus content of parent materials on accumulations of carbon, nitrogen, sulfur and organic phosphorus in grassland soils. Soil Science, v.85, p.307-318. 1958. 